\title{
Capacidad Emprendedora Y Limitantes En La Generación De Emprendimientos: Una Mirada Desde La Condición De Género
}

\author{
Bill Serrano Orellana \\ Luis Brito-Gaona \\ Oscar Riofrío Orozco \\ Guido Sotomayor Pereira
}

Universidad Técnica de Machala - Ecuador

doi: 10.19044/esj.2016.v12n31p96 URL:http://dx.doi.org/10.19044/esj.2016.v12n31p96

\begin{abstract}
Entrepreneurship has a major impact on economic development and poverty reduction; for instance, in developed countries, it has been shown to impact the Gross Domestic Product (GDP) from 5 to 7\%. It is also observed that those who undertake the entrepreneurial challenge tend to invest more in their education, health and welfare in favor of their families, encourage their children to learn to prepare and, thus, promote the desire to grow. Nonetheless, in this 21st century, they still face strong challenges for their full development. For this reason, it is imperative to know the entrepreneurial capacity and the limitations to convert that entrepreneurial capacity into real entrepreneurship. This article shows a statistical analysis of the entrepreneurial capacity by gender. The contribution of this paper is to use the covariance analysis model (ANCOVA) with explanatory variables of reason and dichotomous scales, where the entrepreneurial capacity, measured with Likert-type scales, is the regression. A 20 questions survey was applied fort data compilation. The final objective was to analyze the entrepreneurial capacity between men and women and to determine if there are significant differences among them, as well as the constraints that have blocked the generation of entrepreneurship, even in those individuals with high entrepreneurial capacity. The results show that there are significant differences in entrepreneurship between genders.
\end{abstract}

Keywords: Enterprising capacity, entrepreneurship, gender, limitations of entrepreneurship 


\section{Resumen}

El emprendimiento tiene un impacto importante en el desarrollo económico y la disminución de la pobreza; en países desarrollados, por ejemplo, se ha demostrado que impacta el Producto Interno Bruto (PIB) en alrededor del 5 al 7\%. También se observa que las personas que emprenden tienden a invertir en mayor medida sus ganancias en educación, salud y bienestar a favor de sus familias, fomentan en sus hijos el deseo de aprender a prepararse y tienden a promover el deseo de superación, pero en pleno siglo XXI aún enfrentan fuertes retos para su completo desarrollo. Por ello se hace imperante conocer la capacidad emprendedora y los limitantes para convertir esa capacidad emprendedora en un emprendimiento real. Este artículo presenta un análisis estadístico de la capacidad emprendedora por género. La contribución de este trabajo consiste en utilizar el modelo de análisis de covarianza (ANCOVA) con variables explicativas de escala de razón y dicotómicas, donde la regresada es la capacidad emprendedora medida con escalas de tipo Likert, cuyos datos fueron recopilados mediante la aplicación de una encuesta estructurada por 20 preguntas. El objetivo final fue analizar la capacidad emprendedora entre hombres y mujeres y determinar si existen diferencias significativas, así como los limitantes que han bloqueado la generación de emprendimientos incluso en aquellos individuos con alta capacidad emprendedora. Los resultados demuestran que existe diferencias significativas de la capacidad emprendedora entre géneros.

Palabras claves: Capacidad emprendedora, emprendimiento, género, limitantes del emprendimiento

\section{Introducción}

Mediante el emprendimiento se generan algunos beneficios para la sociedad como son: ampliación de la red empresarial, mejor distribución de la riqueza, disminución de la pobreza, se incrementan los ingresos y se generan nuevas fuentes de trabajo (Leiva, 2008). Nos encontramos en un contexto empresarial donde se evidencia una tendencia creciente relacionada con la creación de negocios por parte de individuos que se ven motivados por distintas razones, tales como: el desempleo, el subempleo, la necesidad de independencia, entre otros. El fenómeno emprendedor es constantemente objeto de análisis para determinar las motivaciones o impedimentos que tienen los emprendedores para empezar su camino empresarial, pero la búsqueda debe ser constante debido a los incesantes y en ocasiones acelerados cambios que sufre el entorno socioeconómico de un determinado sector que se ven afectados tanto por factores internos como externos. Es importante conocer los aspectos que están directamente relacionados con la generación de emprendimientos y el vínculo que existe con otros factores 
que deriven en la generación de nueva y relevante información que permita encontrar soluciones eficientes. Para que se cumpla con todos los propósitos del emprendimiento se necesita al actor principal, el emprendedor, el cual tiene capacidad para la creación de soluciones innovadoras que ayuden al desarrollo económico local, provincial y nacional (Espíritu, González y Alcaraz, 2012).

El emprendedor para iniciar los propósitos del emprendimiento y lograr el éxito, debe tener desarrollada su capacidad emprendedora y conocimientos referente a las actividades que ejecuta. Marulanda, Montoya y Vélez (2014) mencionan que si las personas no cuentan con las capacidades suficientes no se lograrán buenos resultados. Mientras más se conozca la capacidad emprendedora de la persona según su género, nivel socio económico, nivel de instrucción y edad será más fácil fomentar la generación de emprendimientos exitosos. Si bien el emprendimiento forma parte del desarrollo económico del país, conocer más a fondo a los actores que lo hacen posible, desde el estudio exógeno, permitirá propiciar iniciativas que garanticen el éxito, rompiendo barreras que impidan su generación y expansión.

En los últimos años, la mujer ha ido conquistando protagonismo en la sociedad, pasando de ser la cuidadora del hogar a la generadora de ingresos para la familia y participante activa en la economía de un país. Miranda (2012) explica que fue a mediados del siglo XX que se toma en consideración los roles de la feminidad y masculinidad dados por la sociedad. Sin embargo, vivimos en un mundo donde los aspectos biológicos y socioculturales no son distintivos unos de otros (Lamas, 1986). A pesar que la equidad de género en el ámbito empresarial ha tomado mayor relevancia, existen aún varias interrogantes por resolver especialmente en las diferencias de género con la capacidad emprendedora y la intencionalidad de crear una empresa. (Fuentes y Sánchez, 2010).

Con base a lo anterior, el presente trabajo se enfoca en analizar la capacidad emprendedora desde una perspectiva de género; qué tan influyente es la diferencia de género al momento de evaluar la capacidad emprendedora en aquellos que aún no emprenden y los limitantes que impiden la generación de emprendimientos.

\section{Influencia de la condición de género en el emprendimiento}

Gutiérrez (2015) define el emprendimiento como la actitud y capacidad que tiene una persona para enfrentar retos, realizar nuevos proyectos y alcanzar nuevas metas. Es el actuar enfocado en las diversas oportunidades para la generación de valor, solucionando problemas y beneficiando económicamente a la sociedad (Sanabria, Morales y Ortiz, 2015). El emprendimiento es un proceso mediante el cual se generan ideas 
con el fin de crear nuevas empresas asumiendo riesgos en el entorno en el que se desarrolla (Herrera y Gutiérrez, 2014).

Desde un enfoque económico la capacidad emprendedora está relacionada con el rendimiento de la empresa, sector o país, y desde la perspectiva sociológica se centra a rasgos personales (Suárez y Pedrosa, 2016). En este sentido, varios sectores empresariales se han enfocado en el desarrollo de la capacidad emprendedora, en donde se necesita estimular a nivel individual como familiar, experiencias, capacidades que lo diferencien del colectivo (Terán y León, 2010). Entre los rasgos a estimular Omelas, Gonzáles, Olvera y Rodriguez (2015) incluyen la capacidad para la movilización de recursos para la creación de empresas, innovación y la generación de empleo.

Una persona con capacidad emprendedora es racional y se enfoca en realizar de manera eficiente su trabajo bajo la excelente toma de decisiones frente a los beneficios que pueda generar. Es por eso que se lo relaciona con un buen perfil para la creación de empresas (Rusque, 2005). Su capacidad depende de la creencia que tenga de su nivel de conocimiento, habilidades y del entorno en el que se encuentra (Rodríguez y Prieto, 2009). Su manera de actuar se basa en las oportunidades que desea alcanzar (Betancourt Guerrero, 2004).

Por esta razón, Marulanda, Montoya y Vélez (2014) definen a la capacidad emprendedora como la habilidad, destreza y conocimiento que tiene una persona para asumir riesgos y aprovechar las oportunidades de negocios del entorno en el que se desarrolla. Tarapuez y Botero (2007) hacen hincapié en su relación con la capacidad empresarial ya que se enfoca en la creación de empresas. Incluye la creatividad y la orientación a la innovación mediante la planificación, organización y dirección de proyectos enfocados en los objetivos que se desean alcanzar (Tinoco, 2008).

En el campo del emprendimiento se asocian los atributos como el riesgo, creatividad y conquista, siendo esta práctica más masculina, ocasionando así una división del trabajo delegando a las mujeres a cumplir con su rol en el hogar. Un factor importante que distingue la capacidad emprendedora desde la perspectiva de género es la percepción de la oportunidad, en donde, a diferencia de los hombres, las mujeres sienten que tienen esta capacidad (Pineda, 2014). Sin embargo, la falta de confianza y el miedo a fracasar obstaculizan su visión y se limitan a emprender debido al riesgo (Reyes, Pinillos y Soriano, 2014). Además, del rol de la mujer en la familia, delimitando el desarrollo de su capacidad emprendedora (Elizundia, 2015).

Ventura y Quero (2013) mencionan que los hombres son más destacados en la cultura de los negocios y la capacidad que tienen para emprender debido a la absoluta confianza que poseen. Castiblanco (2013) 
indica que el mejor desempeño de la percepción en los hombres se debe a la alta sensibilidad que tienen las mujeres. Aunque en el tema de las motivaciones existe cierta similitud entre hombres y mujeres como son las necesidades de: logro y realización (Sánchez y Fuentes, 2013).

\section{Métodos}

Los datos usados para la presente investigación corresponden a una encuesta realizada a los habitantes de la ciudad de Machala. El cuestionario contiene preguntas que permitieron determinar la capacidad emprendedora del individuo, para ello, se utilizó el instrumento validado por (Flores, 2003). Además, preguntas para determinar: el género, edad, nivel de instrucción y nivel socio económico (se lo determinó utilizando el instrumento establecido por el INEC en el último CENSO realizado en el Ecuador en el año 2010). Para una descripción detallada de todas las variables del modelo véase la Tabla 1.

Tabla 1: Definición y medición de las variables del modelo

\begin{tabular}{|c|c|}
\hline Variable & Definición / medición \\
\hline \multicolumn{2}{|l|}{ Variables dependiente } \\
\hline Capacidad emprendedora & $\begin{array}{l}\text { Habilidad, destreza y conocimiento que tiene la persona } \\
\text { para asumir riesgos y aprovechar las oportunidades de } \\
\text { negocios del entorno en el que se desarrolla basada en } 5 \\
\text { dimensiones: conocimiento de sí mismo, visión del } \\
\text { futuro, motivación de logro, planificación y persuasión. }\end{array}$ \\
\hline \multicolumn{2}{|l|}{ Variables Independientes } \\
\hline Género & Tipo de género de la persona (hombre o mujer) \\
\hline Edad & Edad actual de la persona \\
\hline Nivel de instrucción & $\begin{array}{l}\text { Tipo de educación culminada de la persona (primaria, } \\
\text { secundaria, superior, postgrado) }\end{array}$ \\
\hline Nivel socio económico & $\begin{array}{c}\text { Clase social de la persona (bajo, medio bajo, medio } \\
\text { típico, medio alto, alto) }\end{array}$ \\
\hline
\end{tabular}

\section{Variable Dependiente}

Como se lo mencionó anteriormente para medir la capacidad emprendedora se utilizó el instrumento validado por (Flores, 2003). Esta variable está conformada por cinco dimensiones: 1) conocimiento de sí mismo y autoconfianza, 2) visión de futuro, 3) motivación de logro, 4) planificación y 5) persuasión. Para medir la capacidad emprendedora se asignaron valores en las siguientes proporciones: nunca (0), casi nunca (1), en ocasiones (2), con frecuencia (3), casi siempre (4) y siempre (5), en un total de 20 preguntas. El puntaje máximo que se obtiene de aplicar el instrumento es de 100, de este modo, la variable dependiente se convierte en una variable de escala de razón que indica la capacidad emprendedora.

\section{Variable Independiente}


La principal variable independiente es el género, que se aproximó mediante hombre y mujer. Con el fin de disminuir el potencial de la variable omitida se introdujeron en el estudio variables que son influyentes en la capacidad emprendedora: nivel de instrucción que se establece en una escala de 4 niveles (primaria, secundaria, superior y postgrado), el Nivel socio económico que se genera en 5 grupos (bajo, medio bajo, medio típico, medio alto y alto) y la variable edad con naturaleza en escala de razón.

\section{Marco Econométrico}

Se estimó una ecuación de regresión lineal, donde la variable dependiente es en escala de razón, y se analizó las diferencias desde la condición de género, introducida por una variable dicotómica. Con la misma naturaleza se introdujeron dos de las tres variables que permiten reducir el sesgo de la variable omitida (nivel de instrucción y nivel socio económico).

El modelo de regresión lineal utilizado es ANCOVA que está diseñado cuando los datos a obtener son de respuesta cuantitativa, con variables explicativas cuantitativas y cualitativas. El modelo de regresión es:

$Y_{i}=\beta_{0}+$ i. $\beta_{G e n} \operatorname{Gen}_{I}+\beta_{\mathrm{e}} \operatorname{Edad}_{\mathrm{I}}+$ i. $\beta_{\mathrm{NI}} \mathrm{NI}_{\mathrm{I}}+\mathrm{i} . \beta_{\mathrm{NS}} \mathrm{NS}_{\mathrm{I}}+\mathrm{ui}$

Donde Y denota la capacidad emprendedora. Las otras variables del modelo son: $\mathrm{Gen}_{\mathrm{i}}$ denota el género del individuo; $\mathrm{Edad}_{\mathrm{i}}$ la edad de la persona; $\mathrm{NI}_{\mathrm{I}}$ el nivel de instrucción de la persona; $\mathrm{NS}_{\mathrm{I}}$ el nivel socio económico del individuo.

\section{Limitantes para Generar un emprendimiento}

Para analizar los limitantes para generar emprendimientos, se identificaron los factores causales basados en estudios empíricos, que mediante una escala de Likert (Totalmente en desacuerdo=1, En desacuerdo=2, Neutro=3, De acuerdo=4, Totalmente de acuerdo=5) se obtuvo la puntuación, para luego establecer sus medias. Mediante una comparación de medias (muestras independientes), se determinó si existe diferencias significativas entre hombres y mujeres por cada uno de los limitantes para generar emprendimientos.

\section{Resultados}

Los resultados de la regresión para estimar la capacidad emprendedora según los factores establecidos se presentan en la tabla 2. 
Tabla 2: Resultados principales

\begin{tabular}{|c|c|}
\hline Resultados Principales & Capacidad Emprendedora \\
\hline \multicolumn{2}{|l|}{ Variables Independientes } \\
\hline Género & $\begin{array}{l}-9.436^{* *} \\
(-11.29)\end{array}$ \\
\hline Edad & $\begin{array}{c}0.119 * * \\
(3.11)\end{array}$ \\
\hline \multicolumn{2}{|l|}{ Nivel de instrucción } \\
\hline Secundaria & $\begin{array}{c}5.641 * * \\
(3.13)\end{array}$ \\
\hline Superior & $\begin{array}{c}10.463^{* *} \\
(5.58)\end{array}$ \\
\hline Postgrado & $\begin{array}{c}6.392^{* *} \\
(2.81)\end{array}$ \\
\hline \multicolumn{2}{|l|}{ Nivel socio económico } \\
\hline Medio-bajo & $\begin{array}{c}13.326^{* *} \\
(4.41)\end{array}$ \\
\hline Medio-típico & $\begin{array}{c}17.825^{* *} \\
(5.95)\end{array}$ \\
\hline Medio-alto & $\begin{array}{c}18.950 * * \\
(6.28)\end{array}$ \\
\hline Alto & $\begin{array}{c}20.581 * * \\
(6.32)\end{array}$ \\
\hline Constante & $\begin{array}{c}51.394^{* *} \\
(14.90)\end{array}$ \\
\hline \multicolumn{2}{|l|}{ Índices } \\
\hline $\mathrm{N}$ & 982 \\
\hline$F(9,972)$ & $34.24 * *$ \\
\hline $\mathrm{R}$ - squared & 0.2407 \\
\hline
\end{tabular}

Notas: **, * denotan significancia estadística menor a $1 \%$ y menor a $5 \%$ respectivamente

En lo que respecta al efecto de las variables introducidas para disminuir el potencial de la variable omitida (nivel de instrucción, nivel socioeconómico y edad), los resultados indicaron que sí generan influencia sobre la capacidad emprendedora de forma altamente significativa $(\mathrm{p}<0.01)$. Siendo más específicos, la influencia de la variable nivel de instrucción es altamente significativa $(\mathrm{p}<0.01)$, demostrando que el comportamiento de la capacidad emprendedora es diferente de acuerdo al nivel de instrucción del individuo; el nivel superior es el que mayor cambio generó en la capacidad emprendedora, seguido por postgrado y por último el nivel secundario. El efecto del nivel socio económico fue positivo y altamente significativo $(\mathrm{p}<0.01)$, explicando que la capacidad emprendedora aumenta cuando la persona pertenece a un estrato social más alto. La variable edad también 
denotó alta significancia $(\mathrm{p}<0.01)$, a mayor edad mayor capacidad emprendedora, sin embargo, el efecto generado es ligeramente marginal.

Con base a lo anterior (mejorando la confiabilidad del modelo) los resultados encontrados apoyan la hipótesis que la capacidad emprendedora está influenciada por el género, cuya evidencia es altamente significativa $(\mathrm{p}<0.01)$; la capacidad emprendedora es mayor en el hombre, con una diferencia de 9.43 puntos en relación a la mujer. Esta evidencia es congruente con investigaciones previas (p. ej., Díaz, Hernández, Sánchez y Postigo,2010; Reyes, Pinillos y Soriano, 2014).

A continuación, se detallan las medias de la capacidad emprendedora de cada una de las variables del estudio, sin considerar el efecto entre ellas; también se detalla la calificación cualitativa:

Tabla 3: Calificación cualitativa por Variables diferenciado por género

\begin{tabular}{ccccc}
\hline Capacidad Emprendedora & Hombre & Calificación & Mujer & Calificación \\
\hline Total & 80.71 & Alto & 70.48 & Alto \\
Nivel de instrucción & & & & \\
Primaria & 70.56 & Alto & 60.13 & Mediano \\
Secundaria & 78.13 & Alto & 68.07 & Alto \\
Superior & 84.36 & Alto & 73.69 & Alto \\
Postgrado & 80.37 & Alto & 72.45 & Alto \\
Nivel Socio económico & & & & \\
Bajo & 57.4 & Mediano & 51.06 & Mediano \\
Medio-bajo & 71.53 & Alto & 67.28 & Alto \\
Medio-típico & 81.54 & Alto & 71.46 & Alto \\
Medio-alto & 82.78 & Alto & 72.67 & Alto \\
Alto & 85.27 & Muy Alto & 74.28 & Alto \\
\hline
\end{tabular}

Los limitantes para generar emprendimientos se detallan en la tabla 4. Tabla 4: Limitantes para generar un emprendimiento

\begin{tabular}{|c|c|c|c|c|c|c|}
\hline \multirow{2}{*}{ LIMITANTES } & \multicolumn{2}{|c|}{ Mujer } & \multicolumn{2}{|c|}{ Hombre } & \multicolumn{2}{|c|}{ Comparación de Medias } \\
\hline & Media & SD & Media & SD & $\mathrm{T}$ & $\mathrm{p}$ \\
\hline Escaso capital para iniciar la empresa & 4.18 & 0.88 & 4.30 & 2.72 & -0.640 & 0.524 \\
\hline $\begin{array}{c}\text { Insuficiente apoyo de entidades } \\
\text { públicas y privadas para crear la } \\
\text { empresa }\end{array}$ & 3.76 & 1.08 & 3.73 & 1.13 & 0.350 & 0.726 \\
\hline $\begin{array}{c}\text { Baja confianza en sus capacidades } \\
\text { emprendedoras }\end{array}$ & 2.93 & 1.34 & 2.73 & 1.35 & 0.081 & 0.000 \\
\hline $\begin{array}{l}\text { No tiene bien en claro que empresa } \\
\text { va a crear }\end{array}$ & 2.79 & 1.22 & 2.84 & 1.31 & -0.400 & 0.691 \\
\hline $\begin{array}{c}\text { Falta de socios para iniciar una } \\
\text { empresa }\end{array}$ & 3.15 & 1.26 & 3.13 & 1.30 & 0.140 & 0.885 \\
\hline $\begin{array}{l}\text { No se producir ni fabricar nada en } \\
\text { particular }\end{array}$ & 2.60 & 1.33 & 2.65 & 0.42 & -0.590 & 0.599 \\
\hline
\end{tabular}

El limitante para generar un emprendimiento que mayor puntuación obtuvo tanto en hombres y mujeres fue "Escaso capital para iniciar una 
empresa”, con una ligera superioridad en el hombre, sin embargo, la diferencia no fue significativa $(\mathrm{p}=0.524)$. Por otro lado, el limitante que menor puntuación obtuvo fue "No se producir nada en particular", acentuándose en el hombre ligeramente, cuya diferencia no fue significativa $(p=0.590)$.

\section{Discusión}

El objetivo de este trabajo fue establecer si la capacidad emprendedora está determinada por la condición de género. Él estudió se efectuó de una manera dual, analizando la capacidad emprendedora y diferenciándola por condición de género y determinando los limitantes para generar un emprendimiento con un análisis por condición y entre géneros, lo que permite comprobar si existe alguna sensibilidad de las variables estudiadas para corroborar la hipótesis planteada. Además, el presente trabajo a diferencia de otros existentes en la literatura, mide el impacto de factores exógenos en la capacidad emprendedora y no se centra en los factores endógenos, lo que permitió obtener información de mayor valor para el ámbito práctico.

De los resultados obtenidos se observó que dentro del constructo condición de género existen diferencias significativas en cuanto a su capacidad emprendedora, siendo ésta mayor en el hombre, confirmando lo manifestado por el estudio empírico realizado por (Reyes, Pinillos y Soriano, 2014). Lo que nos permite también establecer que los hombres tienen mayor garantía de éxito al generar un emprendimiento, ya que tener mayor capacidad emprendedora es sinónimo de mayores habilidades que permitan solucionar problemas (Moreno, 2002), mayor creatividad, asunción de riesgos, necesidad de logro, autonomía y determinación (Rusque 2005), y aprovechamiento de oportunidades (Marulanda, Montoya y Vélez, 2014).

En lo que respecta a las variables introducidas para disminuir el potencial de la variable omitida (nivel de instrucción, nivel socio-económico y la edad), estas afectan positiva y significativamente la capacidad emprendedora. Aquellas personas con nivel de instrucción superior o universitario tienen la mayor capacidad emprendedora, seguido por postgrado, secundaria y primaria. En cuanto al nivel socio económico, a mayor nivel, mayor capacidad emprendedora. La edad también genera el mismo efecto incremental pero ligeramente marginal.

De los resultados derivados del análisis de los limitantes para generar un emprendimiento, se establece que la mayor limitante en los hombres y mujeres fue "escaso capital para iniciar la empresa", sin embargo, la diferencia entre ellos no fue significativa, por otro lado, el limitante de menor impacto fue "no se producir ni fabricar nada”, siendo sus diferencias no significativas. 
En cuanto a las implicaciones prácticas del presente trabajo, se deriva la ventaja de conocer cuáles son los factores exógenos que influyen en la capacidad emprendedora para dos contextos importante en la economía de un país: 1) Gerencial. Las empresas persiguen crecer y una de las vías para conseguirlo es a través de nuevos emprendimientos. Por lo tanto, conocer quiénes son los actores con mayor capacidad emprendedora puede garantizar el éxito de este nuevo desafío. 2) Política Pública. Desde las instituciones y empresas públicas y conscientes de la importancia que tiene para el conjunto de la economía los emprendimientos, existe una serie de programas para impulsar la generación de estos. En este sentido el mejor conocimiento de aquellos actores que tienen mayor capacidad emprendedora, ayudará a una mejor instrumentalización de estos apoyos al canalizar estas hacia aquellos que potencien los emprendimientos.

\section{References:}

1. Betancourt Guerrero, B. (2004). La capacidad emprendedora y el desarrollo de nuevas empresas. Cuaderno de Administración, 20(32), 107-136.

2. Castiblanco Moreno, S. E. (2013). La construcción de la categoría de emprendimiento femenino. Revista Facultad de Ciencias Económicas: Investigación y Reflexión, 21(2), 53-66.

3. Díaz, J., Hernández, R., Sánchez, M., \& Postigo, M. (2010). Actividad emprendedora y género. Un estudio comparativo. Revista Europea de Dirección y Economía de la empresa, 19(2), 83-98.

4. Elizundia Cisneros, M. E. (2015). Desempeño de nuevos negocios: perspectiva de género. Contaduría y Administración, 60(2), 468-485.

5. Espíritu, R., González, R., \& Alcaraz, E. (2012). Desarrollo de competencias emprendedoras: Un análisis explicativo con estudiantes universitarios. Cuadernos de Estudios Empresariales, 22(22), 29-53.

6. Fuentes, F., \& Sánchez, S. (2010). Análisis del perfil emprendedor: una perspectiva de género. Estudios de Economía Aplicada, 28(3), 128.

7. Gutiérrez, S. (2015). Emprendimiento en las empresas familiares. Revista Iberoamericana de Contaduría, Economía y Administración:RICEA, 4(7), 149-167.

8. Herrera, K., \& Gutiérrez, J. (2014). El emprendimiento como iniciativa para la creación de empresas: análisis de la perspectiva psicológica y contextual. Revista de Ciencias Sociales RCS, 20(2), 288-302.

9. Lamas, M. (1986). La antropología feministista y la categoría género. Nueva antropología, 9(30), 174-198. 
10. Leiva, J. C. (2008). ¿Nacen empresas de las actividades de fomento al espiritu emprendedor? TEC Empresarial, 2(1), 16-27.

11. Marulanda, F., Montoya, I., \& Veéez, J. (2014). Aportes teóricos y empíricos al estudio del emprendedor. Cuadernos de Administración, 30(51), 89-99.

12. Miranda Novoa, M. (2012). Diferencia entre la perspectiva género y la ideologia género. DIKAION, 21(2), 337-356.

13. Moreno, J. (2002). Capacidad emprendedora en la educacion superior. Balance de la experiencia del servicio de atención al emprendedor(SAE) en la universidad metropolitana. ANALES, 2(2), 149-160.

14. Omelas, C., Gonzáles, L., Olvera, M \& Rodriguez, M. d. (2015). El Espíritu Emprendedor y un factor que influencia su Desarrollo Temprano. Conciencia Tecnológica(49), 46-51.

15. Pineda Duque, J. (2014). Emprendimiento y género: el caso de la industria de la belleza en Bogotá. Sociedad y Economía(26), 239-272.

16. Reyes, L., Pinillos, M. J., \& Soriano, I. (2014). Diferencias de género en la orientación emprendedora. Market Economics and Business Journal, 45(3), 441-460.

17. Rodríguez, C., \& Prieto, F. (2009). La sensibilidad al emprendimiento en los estudiantes universitarios. Estudio comparativo Colombia-Francia. INNOVAR. Revista de Ciencias Administrativas y Sociales, 19(1), 73-89.

18. Rusque, A. M. (2005). Capacidad emprendedora y capital social. Revista venezolana de análisis de coyuntura, 11(2), 189-202.

19. Sanabria, S., Pedraza, P., \& Hurtado, E. (2014). El emprendimiento como fuente de desarrollo y fortalecimiento de las capacidades endógenas para el aprovechamiento de las energías renovables. EAN.Escuela de Administración de negocios Institución Universitaria(77), 152-167.

20. Sánchez, S., \& Fuentes, F. (2013). Mujer y emprendimiento: Un análisis en el contexto universitario español. Revista de Ciencias Sociales RCS, 19(1), 140-153.

21. Suárez, j., \& Pedrosa, I. (2016). Evaluación de la personalidad emprendedora: situación actual y líneas de futuro. Papeles del Psicólogo, 37(1), 62-68.

22. Tarapuez, E., \& Botero, J. (2007). Algunos aportes de los neoclásicos a la teoría del emprendedor. Cuaderno de Administración, 20(34), 39-63.

23. Terán, A., \& León, G. (2010). Visión del emprendimiento desde el estado y la universidad: El plan de desarrollo de Antioquia visión 
2020 con el programa de emprendimiento de la UPB. Revista Ciencias Estratégicas, 18(23), 119-129.

24. Tinoco Gómez, O. (2008). Medición de la Capacidad Emprendedora de ingresantes a la Facultad de Ingenieria Industrial de la UNMSM. Industrial Data. Revista de Investigación, 11(2), 18-23.

25. Ventura, R., \& Quero, M. J. (2013). Factores explicativos de la intención de emprender en la mujer. Aspetos diferenciales en la población universitaria según la variable género. Cuadernos de Gestión, 13(1), 127-149. 